\title{
ULTRASOUND AS A TOOL FOR ENDOTRACHEAL TUBE CONFIRMATION IN PATIENTS WITH OR WITHOUT DIFFICULT AIRWAY UNDERGOING ELECTIVE SURGERY
}

\author{
Nidhi Rani Gupta1, Deepa George2, Letha J. Babukutty³, Vimal Pradeep ${ }^{4}$ \\ ${ }^{1}$ Lecturer, Department of Anaesthesia, Government T. D. Medical College and Hospital, Alappuzha, Kerala. \\ ${ }^{2}$ Assistant Professor, Department of Anaesthesia, Government T. D. Medical College and Hospital, Alappuzha, Kerala. \\ ${ }^{3}$ Associate Professor, Department of Anaesthesia, Government T. D. Medical College and Hospital, Alappuzha, Kerala. \\ ${ }^{4}$ Assistant Professor, Department of Anaesthesia, Government T. D. Medical College and Hospital, Alappuzha, Kerala.
}

\section{ABSTRACT}

\section{BACKGROUND}

Aims- Ultrasound is underutilised but effective tool for airway evaluation and management in emergency and elective conditions. In this study, primary aim was to compare the confirmation time for endotracheal intubation by USG versus waveform capnography in patients with MPG I - IV. Secondary aim was to calculate sensitivity, specificity and accuracy of using ultrasound for confirmation of endotracheal tube placement.

\section{MATERIALS AND METHODS}

A prospective, single centre, observational study was carried out in patients undergoing elective surgery and requiring endotracheal intubation. A total of 50 patients from each MPG were recruited making a total of 200. During intubation, real time transtracheal ultrasonography was performed to visualise the passage of endotracheal tube. Bag ventilation was started, capnography with end tidal $\mathrm{CO}_{2}$ levels was observed by other faculty and simultaneous auscultation of chest and epigastrium was done. Bilateral lung USG was done for detection of lung sliding as an indicator of bilateral lung ventilation, which was finally confirmed by subxiphoid view, which showed diaphragmatic movement.

\section{RESULTS}

1. USG was better than waveform capnography in terms of confirmation time in all 4 groups $(\mathrm{p}<0.001)$.

2. Transtracheal ultrasonography had $96 \%$ accuracy for confirmation of endotracheal intubation.

3. Overall sensitivity was $100 \%$ and specificity of MP I - III was more than $80 \%$.

4. Overall specificity of MPG IV was $25 \%$, but on subgroup analysis into patients with or without distorted neck anatomy, specificity was more than $80 \%$ in patients with normal neck anatomy.

\section{CONCLUSION}

Ultrasound is a rapid and accurate tool for confirmation of endotracheal tube placement in patients with or without difficult airway undergoing elective surgery but visualisation of trachea is difficult in cases with distorted neck anatomy.

\section{KEYWORDS}

Mallampati Grading (MPG), Ultrasonography (USG), Capnography.

HOW TO CITE THIS ARTICLE: Gupta NR, George D, Babukutty LJ, et al. Ultrasound as a tool for endotracheal tube confirmation in patients with or without difficult airway undergoing elective surgery. J. Evolution Med. Dent. Sci. 2017;6(39):3124-3127, DOI: 10.14260/Jemds/2017/673

\begin{abstract}
BACKGROUND
Endotracheal intubation is an essential step for airway management and adequate ventilation during various surgical procedures. Oesophageal or main stem intubation are major complications associated with this procedure, which if not timely detected, can lead onto life-threatening consequences. There are various direct and indirect methods to confirm the correct placement of the endotracheal tube: passage of the tube through vocal cords under direct visualisation, auscultation of bilateral chest for presence of equal breath sounds and epigastrium for absence of gurgling sound, bilateral equal chest movement on bag ventilation,
\end{abstract}

Financial or Other, Competing Interest: None.

Submission 10-04-2017, Peer Review 03-05-2017,

Acceptance 10-05-2017, Published 15-05-2017.

Corresponding Author:

Nidhi Rani Gupta,

Lecturer, Department of Anaesthesia,

J-Block, TDMCH, Vandanam-688005,

Alappuzha, Kerala.

E-mail: drnidhigarg@gmail.com

DOI: $10.14260 /$ jemds $/ 2017 / 673$

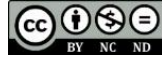

fibreoptic bronchoscopy, pulse oximetry and qualitative and/or quantitative end-tidal CO2 measurement.

The policy statement was published by American College of Emergency Physicians on the verification of endotracheal intubation writing ultrasound as a possible future adjunct. ${ }^{1}$ Use of ultrasound in airway management has been extended to critically ill patients due to its low cost and portability. ${ }^{2}$ Ultrasound confirm endotracheal intubation in a number of ways, i.e. scanning at the level of the cricoid membrane in which direct visualisation of the passage of the endotracheal tube through the vocal cords is possible. ${ }^{3}$ After intubation, ultrasonography just above the level of suprasternal notch, if oesophageal shadow is absent indicates tracheal intubation indirectly. ${ }^{4}$ Real-time visualisation of endotracheal tube placement may be possible by transtracheal scanning just below the cricoid ring. ${ }^{4,5}$ During bag ventilation in intubated patients- bilateral lung sliding and diaphragmatic excursions as visualised by using low frequency curvilinear probe has been shown to be both sensitive and specific for verification of endotracheal tube position. ${ }^{3}$ If lung sliding is absent over one lung indicates either main stem intubation or pneumothorax and if absent over both lungs then either 
oesophageal intubation or cuff leak present. Very few studies have been done till date regarding the confirmation of tube placement during the intubation only using ultrasound as a modality in live patients undergoing elective surgery.

In this study, primary objective was to compare the ultrasound $\mathrm{v} / \mathrm{s}$ waveform capnography for tube either tracheal or oesophageal confirmation in patients with I - IV MPG. Secondary objective was to calculate overall accuracy, sensitivity and specificity (MPG - I - IV) of USG for confirmation of tube placement.

\section{MATERIALS AND METHODS}

A prospective, single centre, observational study was done in patients between 18 - 55 years of either gender, who underwent elective surgery with endotracheal intubation in Govt. TDMCH, Vandanam, Alappuzha between April 2016 and November 2016 after ethical clearance from the institute and consent was taken from every patient. Patient was intubated with the help of direct laryngoscopy and endotracheal tube and stylet was used if required.

During intubation, the transducer (High frequency 10 $15 \mathrm{MHz}$ linear probe, Mindray Machine) was placed just above the suprasternal notch for transtracheal real-time (Transverse view) ultrasonography for direct visualisation of passage of the endotracheal tube into the trachea or oesophagus. The endotracheal tube position was defined as tracheal intubation if only one A - M interface with comet-tail artefact and posterior shadowing was there. Oesophageal intubation was defined if two A - M interfaces with comet-tail artefacts and posterior shadowing was there, which is known as a "double tract sign."6 Immediately bag ventilation was started, other faculty observed for waveform capnography and ETCO2 level and simultaneously auscultation of chest and epigastrium was also done. The bilateral lung ultrasound was done for detection of lung sliding at visceral parietal pleural surface as indication of bilateral lung ventilation, which was finally confirmed by observing diaphragmatic movement by subxiphoid view with ultrasound. Low frequency curvilinear $4-5 \mathrm{MHz}$ probe was used for lung and diaphragmatic ultrasound. Ultrasonography in all cases was done by faculty having a minimum experience of 2 years. All intubations were performed by $2^{\text {nd }}$ and $3^{\text {rd }}$ year junior residents.

\section{Ethics}

The study was conducted after obtaining approval from Institutional Research and Ethical Committee and written informed consent was taken from all patients. The procedures followed were in accordance with the ethical standards of the responsible committee on human experimentation (Institutional or Regional) and with the Helsinki Declaration of 1975 that was revised in 2000.

\section{Statistics}

Statistical analysis was done using SPSS version 21, independent ' $\mathrm{t}$ ' test.

Confirmation time for both groups was calculated as Mean \pm S.D. and compared by ' $p$ ' value. Sensitivity and specificity, positive and negative predictive value was also calculated for all the subjects and the subgroups.

\section{Sample Size}

Based on previous studies done by Sustic et al(7) and Rajan S et al(8) based on results of sensitivity of using ultrasound with $95 \%$ confidence interval and $20 \%$ allowable error the minimum sample size was calculated as 40 .

But to increase the sensitivity and specificity of our study, we had selected a total of 200 patients which was further segregated into 4 groups of 50 each according to MPG I - IV. As the incidence of MPG III - IV is less than MPG I - II, but to make the 4 groups comparable and to avoid selection bias, 50 equal patients were recruited into each group.

\section{RESULTS}

A sample of 200 patients were recruited, divided into equal 4 groups according to MPG I - IV. Confirmation time was started from when the laryngoscope blade was introduced into the mouth up to confirmation of intubation by transtracheal ultrasonography or capnography (Table 1, Figure 1).

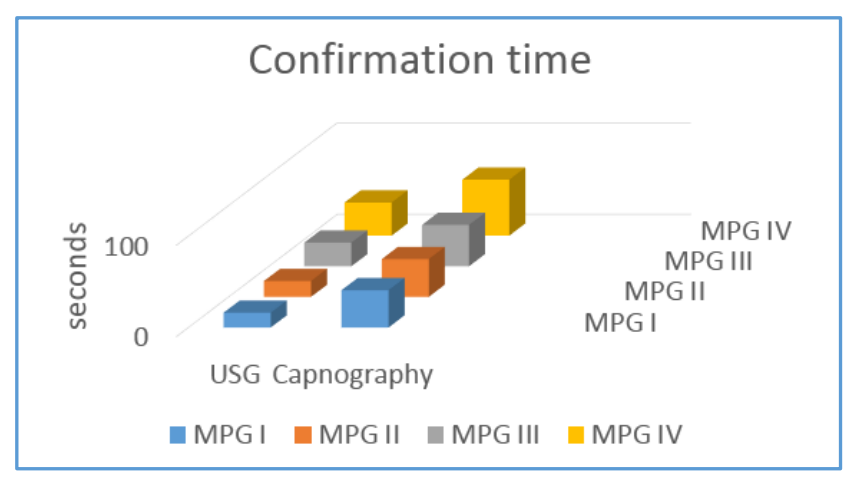

\begin{tabular}{|c|c|c|c|c|}
\hline Seconds & MPG - I & MPG - II & MPG - III & MPG - IV \\
\hline $\begin{array}{c}\text { USG } \\
\text { (Mean } \pm \text { SD) }\end{array}$ & $16.24 \pm 5.45$ & $17.46 \pm 8.28$ & $25.82 \pm 11.80$ & $36.2 \pm 9.56$ \\
\hline Capnography & $40.84 \pm 6.28$ & $41.32 \pm 8.14$ & $45.24 \pm 12.42$ & $61.18 \pm 11.72$ \\
\hline P value & $<0.001$ & $<0.001$ & $<0.001$ & $<0.001$ \\
\hline Table 1. (Confirmation Time of Tracheal Intubation) \\
\hline
\end{tabular}

Sensitivity and specificity along with positive and negative predictive value of ultrasonography for confirmation of endotracheal tube in MPG I - IV was calculated. In case of MPG - I and II, both USG and capnography confirmed tracheal intubation in 49 patients out of 50 . Hence sensitivity, specificity, PPV and NPV was 100\%. In MPG - III USG identified 45 out of 50 intubations as tracheal, whereas capnography confirmed only 44 . Hence, sensitivity was $100 \%$, specificity $83 \%$, PPV $97 \%$ and NPV $100 \%$.

In MPG - IV, USG identified 48 out of 50 intubations as tracheal, whereas capnography confirmed only 42 . Hence, sensitivity was $100 \%$, specificity $25 \%$, PPV $87 \%$ and NPV $100 \%$. Thus, to confirm the reason behind this, the group was further segregated into two subgroups according to neck anatomy, i.e. normal, distorted (big neck swelling, contracture neck, injury to neck) cases in which visualisation of trachea by USG was difficult; 35 had normal and 15 had distorted neck anatomy. In normal neck patients, USG and capnography confirmed 33 out of 35 intubations as tracheal. Hence, sensitivity, specificity, PPV and NPV was $100 \%$. In patients with distorted neck anatomy, USG identified 14 out of 15 intubations as tracheal, whereas capnography confirmed only 9. Hence, sensitivity was $100 \%$, specificity $16 \%$, PPV $64 \%$ and 
NPV 100\%. Overall, USG identified 191 out of 200 intubations as tracheal, whereas capnography confirmed only 184 . Hence, sensitivity was $100 \%$, specificity 56\%, PPV 96\%, NPV 100\% and accuracy $96 \%$.

If distorted neck anatomy patients (MPG IV - b) excluded, USG identified 176 out of 185 intubations as tracheal, whereas capnography confirmed only 175 . Hence, sensitivity was $100 \%$, specificity $90 \%$, PPV $99 \%$ and NPV $100 \%$.

\section{DISCUSSION}

Tracheal intubation is one of the essential step for smooth and successful conduction of surgery under general anaesthesia. Thus, the confirmation of endotracheal intubation is of paramount importance to avoid lifethreatening complications. Variety of direct and indirect methods are used for the confirmation of endotracheal intubation. Ultrasonography is a real time, quick, portable, novel tool in which there is no need for ventilation and can be used in patients in cardiac arrest and absent pulmonary blood flow. It can confirm endotracheal intubation by direct and indirect methods. Indirect methods are- a) Bilateral lung sliding sign (Visceral parietal pleura interface) ${ }^{7-10}$ and b) Diaphragmatic excursion (Subxiphoid view). ${ }^{9-11}$

Direct methods are- a) Sagittal view- Long axis view of either trachea or paratracheal space; b) Transcricoid viewWidening of vocal cords during passage of tube. ${ }^{3}$ This view is mainly used in paediatric patients for confirmation; and c) Transtracheal (Transverse view)- Visualisation of trachea and oesophagus both at the same time during intubation..$^{4,5}$ This transtracheal view was used in this study and found to be an appropriate view for confirmation of intubation as both trachea and oesophagus were visualised simultaneously.

In this study, we took a sample of 200 patients divided into 4 equal groups according to MPG I - IV. Our primary outcome was the comparison of confirmation time and we found that the ultrasonography took statistically significant less time for confirmation in all the 4 groups than waveform capnography. Our results are in concordance with Chou $\mathrm{H}$ et al $^{6}$ They also found as TRUE (Tracheal rapid ultrasound exam) can be performed rapidly than capnography in emergency intubation. Another study done by Das SK et al ${ }^{12}$ also concluded that ultrasound as a rapid and reliable tool for confirmation than capnography. Accuracy was found to be $96 \%$ for using ultrasound for confirmation. Our secondary outcome was to calculate sensitivity and specificity along with positive and negative value for ultrasound as a tool for confirmation of intubation as overall and differently for 4 groups. We found that sensitivity and specificity in MPG I - II was $100 \%$. In MPG III specificity is $83 \%$, but however in MPG IV it is decreased to $25 \%$. Thus, to evaluate the cause for this discrepancy, we segregated the MPG IV group into further 2 sub-groups according to neck anatomy- a) Normal, b) Distorted. We found that if we exclude the MPG IV - b group, again specificity came out to be $100 \%$. These findings are in concordance with $\mathrm{Chou} \mathrm{H}$ et $\mathrm{al}^{13}$ who did meta-analysis and found $93 \%$ sensitivity and $97 \%$ specificity for detection of oesophageal intubation by ultrasonography. Similar results were observed by Gottlieb et al ${ }^{14}$ that $98 \%$ sensitivity and $98 \%$ specificity for ultrasonographic transtracheal confirmation of intubation.
The limitations of our study were firstly, we included patients who underwent elective surgery and emergency intubations were not a part of the study. Thus, the results cannot be applied for emergency procedures. Secondly, difficult airway was classified according to Mallampati grading and no other parameters were included. Thirdly, patients with difficulty in visualisation of trachea due to abnormal neck anatomy were part of the study with leads onto such a huge discrepancy in results for MPG IV.

\section{CONCLUSION}

Thus, we conclude that ultrasound is a rapid and accurate tool with high sensitivity and specificity for confirmation of endotracheal tube placement in patients with or without difficult airway undergoing elective surgery, but visualisation of trachea is difficult in cases with distorted neck anatomy.

\section{REFERENCES}

[1] American College of Emergency Physicians. Clinical Policy Committee. Verification of endotracheal tube placement. Ann Emerg Med 2009;54(1):141-2.

[2] Sustic' A. Role of ultrasound in the airway management of critically ill patients. Crit Care Med 2007;35(5 Suppl):S173-7.

[3] Park SC, Ryu JH, Yeom SR, et al. Confirmation of endotracheal intubation by combined ultrasonographic methods in the emergency department. Emerg Med Australas 2009;21(4):293-7.

[4] Werner SL, Smith CE, Goldstein JR, et al. Pilot study to evaluate the accuracy of ultrasonography in confirming endotracheal tube placement. Ann Emerg Med 2007;49(1):75-80.

[5] Drescher MJ, Conard FU, Schamban NE. Identification and description of esophageal intubation using ultrasound. Acad Emerg Med 2000;7(6):722-5.

[6] Chou HC, Tseng WP, Wang $\mathrm{CH}$, et al. Tracheal rapid ultrasound exam (T.R.U.E.) for confirming endotracheal tube placement during emergency intubation. Resuscitation 2011;82(10):1279-84.

[7] Sustic A, Protic A, Cicvaric T, et al. The addition of a brief ultrasound examination to clinical assessment increases the ability to confirm placement of doublelumen endotracheal tubes. J Clin Anesth 2010;22(4): 246-9.

[8] Rajan S, Surendran J, Paul J, et al. Rapidity and efficacy of ultrasonographic sliding lung sign and auscultation in confirming endotracheal intubation in overweight and obese patients. Indian J Anaesth 2017;61(3): 230-4.

[9] Chun R, Kirkpatrick AW, Sirois M, et al. Where's the tube? Evaluation of hand-held ultrasound in confirming endotracheal tube placement. Prehosp Disaster Med 2004;19(4):366-9.

[10] Hsieh KS, Lee CL, Lin CC, et al. Secondary confirmation of endotracheal tube position by ultrasound image. Crit Care Med 2004;32(Suppl 9):S374-S7.

[11] Weaver B, Lyon M, Blaivas M. Confirmation of endotracheal tube placement after intubation using the ultrasound sliding lung sign. Acad Emerg Med 2006;13(3):239-44. 
[12] Das SK, Choupoo NS, Haldar R, et al. Transtracheal ultrasound for verification of endotracheal tube placement: a systematic review and meta-analysis. Can J Anaesth 2015;62(4):413-23.

[13] Chou EH, Dickman E, Tsou PY, et al. Ultrasonography for confirmation of endotracheal tube placement: a systematic review and meta-analysis. Resuscitation 2015;90:97-103.
[14] Gottlieb M, Bailitz JM, Christian E. et al. Accuracy of novel ultrasound technique for confirmation of endotracheal intubation by expert and novice emergency physicians. West J Emerg Med 2014;15(7):834-9. 